\title{
Thoughts on the Emergency Guarantee of Library Electronic Resources in the Post-Pandemic Era
}

\author{
Yan Jiang* \\ Zhongyuan Institute of Technology Library, Zhengzhou 450007, Henan Province, China \\ *Corresponding author: Yan Jiang, 573634575@qq.com \\ Copyright: ( 2022 Author(s). This is an open-access article distributed under the terms of the Creative Commons Attribution License (CC \\ BY 4.0), permitting distribution and reproduction in any medium, provided the original work is cited.

\begin{abstract}
In the post-pandemic era, libraries need to improve the emergency support of electronic resources and establish a relatively perfect electronic resource support system in order to adapt to the construction goal of the national emergency system as well as realize their own transformation and development. It must be realized through enhancing awareness and planning, strengthening policy support and top-level design, realizing rapid response and intelligent response, improving technical support, promoting resource synergy, integration, and sharing, strengthening community integration, as well as providing intelligent management and services.
\end{abstract}

Keywords: Electronic resources; Emergency guarantee; Collaborative integration; Sharing of resources; Intelligent management and services

Online publication: January 18, 2022

\section{Introduction}

What is needed in emergencies is not only the information resources in key emergency areas. In view of the outbreak of COVID-19 in the later part of 2019, adults and children can only stay at home during that period. However, when class stops, learning cannot stop. From education and teaching to various scientific research activities, as well as e-government, public propaganda, and other fields need libraries to provide electronic resource services. All libraries responded quickly, strengthened the integration and provision of electronic resources, and did a lot of information resource emergency support for online teaching, scientific research, academic and government decision-making, think tanks, and so on during the epidemic. However, the ecological development of the library's own electronic resource system is imperfect, especially in the post-pandemic era with the normalization of prevention and control. Traditional literature resources and space services have lost their advantages. How the library should transform and develop is worthy of indepth study.

The General Office of the State Council issued the National Emergency Response System Construction "13th Five-Year Plan" in 2007. The construction goal is to comprehensively strengthen the emergency support ability, improve the social collaborative response ability, and further improve the emergency management system. In 2020, it was pointed out that the fight against COVID-19 should focus on both scientific research and material production. Under this social background, libraries urgently need to improve their emergency service mechanism, establish a relatively perfect electronic resource guarantee system, improve the emergency guarantee ability of electronic resources, and provide services for teaching as well as scientific research in emergencies. 


\section{Improving the emergency support capacity of electronic resources}

An accepted definition of emergency support capability is that it is the comprehensive application of organizational system, emergency plan, technology, resource guarantee, and social mobilization in response to emergencies ${ }^{[1]}$. Based on previous research, it is believed that the emergency support ability of library electronic resources should be a perfection based on the relationship among the subjects, environment, and resources in the supply chain. It includes resources, system platform, network infrastructure, talent cultivation, mechanism, etc. The work of library electronic resources includes the purchase and collection of electronic resources, reserve and application, integrated supply, management service, supervision and evaluation, etc. The library's emergency support capability should closely focus on the supply chain and related work of electronic resources as well as strengthen the coordination and integration of electronic resources, intelligent management and service, technology, platform, as well as personnel.

In the post-pandemic era, as the organizer, coordinator, manager, and service provider in the emergency electronic resource supply chain, the library is limited by adverse conditions, such as the decrease in resource construction funds, the proliferation of online users, the improvement of requirements for technology and facilities, etc. Converting the adverse conditions into favorable conditions and actively adapting to the environment are very important. Only by combining the specific requirements of national prevention and control as well as grasping the main factors can a powerful electronic resources emergency support system be established. Specifically, it is a trinity system with system guarantee, main structure, and emergency service centered on the construction and guarantee of electronic resources ${ }^{[2]}$. The framework of the electronic resources emergency support system constructed in this paper is shown in Figure 1. In this system, all social forces, including the government, enterprises, libraries, communities, and individuals, need to play a role in order to achieve an orderly and efficient emergency management. In terms of libraries, it is necessary to strengthen accurate procurement, development, and integration of electronic resources, optimize service content, as well as improve remote service ability. It is also imperative to upgrade the intelligent management service platform, realize metadata cross system sharing, big data processing, knowledge discovery, and knowledge link, in addition to actively carry out alliance cooperation as well as collaborative integration and sharing of information resources based on the electronic resource supply chain.

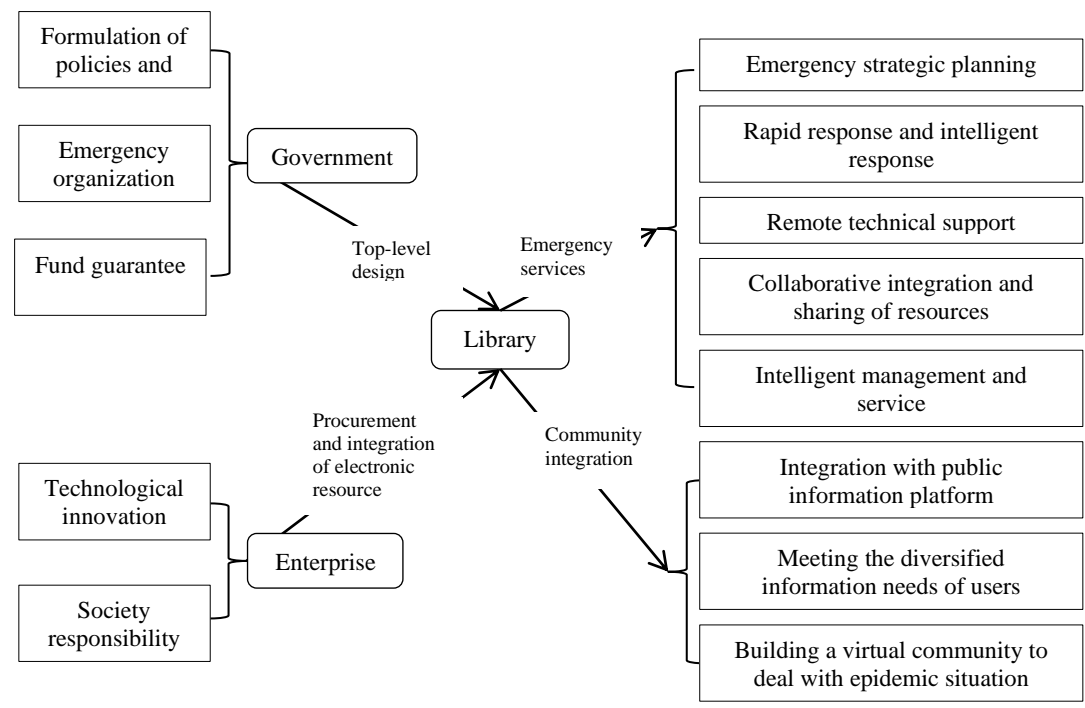

Figure 1. Framework of the library resource emergency support system

\section{Realizing rapid response and intelligent response}

In the post-pandemic era, in order to improve the response to emergencies, libraries need to improve the 
rapid response and institutional response to emergencies, form the concept of normalized emergency support, strengthen the awareness of risk prevention and safety, as well as build a flawless emergency support and management system. Strengthening the revision of relevant laws and regulations, policies, systems, strategic planning, and emergency plans needs to be carried out from multiple levels: at the government level and the library level.

\subsection{Measures at the government level}

In the Law of the People's Republic of China on Public Libraries (2018) and the Regulations on Libraries of Ordinary Institutions of Higher Learning (2015), there are several provisions and contents related to the library's handling of emergencies and pandemic prevention. Emergency protection measures were lacking for all libraries in the face of COVID-19. The contents and standards of online services were not uniform, and the network security and public opinion management were not experienced. The main body of collaborative emergency management should be the government. The relevant state organs should formulate laws, regulations, and articles according to the characteristics of the library to standardize the standards and requirements of emergency support services. Local governments should carry out overall design from a top-level perspective and establish perfect emergency guarantee mechanism, linkage mechanism, and information sharing mechanism ${ }^{[3]}$, which include emergency organization setting, fund management, monitoring and early warning, information submission, as well as emergency plan ${ }^{[3]}$. It is also necessary to strengthen the cooperation of organizations inside and outside the library, establish an emergency information service platform, improve the effectiveness of emergency linkage, promote the rapid concentration and use of important electronic resources at home and abroad, as well as strive for resources to reach the grid community.

\subsection{Measures at the library level}

Firstly, libraries should formulate an emergency support service system and clarify the objectives, tasks, key contents, organizational structure, and coordination mechanism of emergency support service through the system ${ }^{[4]}$. As an important task, resource emergency support is included in daily work deployment and future development planning. In addition to the daily preparation, training, and drill of emergency work process (emergency plan), as well as the reserve and guarantee of resources, it is also necessary to be forward-looking, ensure strategic thinking, and formulate strategic planning for emergency management of electronic resources. The content of the plan should be integrated with the sustainable development goal, which not only needs to improve the quality of electronic resources, but also to deepen the application ${ }^{[5]}$. It includes business architecture, application architecture, data architecture, technical architecture, etc. ${ }^{[5]}$. It is also necessary to build and improve a cross domain collaborative electronic resource intelligent service platform, integrate knowledge resources distributed in different systems, allocate resources scientifically and reasonably, as well as establish an effective resource discovery mechanism and recommendation mechanism relying on the service platform and information environment ${ }^{[6]}$. Other than that, it is imperative to actively participate in and develop knowledge sharing and academic open access resources at home and abroad. Under the guarantee of security mechanism, open data will be processed to build a knowledge base and think tank for the integration of public data and government data.

As the emergency management gradually develops in the direction of data, intelligence, and even ecology, the emergency support work of the library needs to realize the intelligent decision-making of experts, joint and coordinated response, intelligent management and service, as well as assume measures according to the crisis level. The libraries should provide users with electronic resources with more openness, timeliness, stability, standardization, intelligence, and other characteristics, as well as use multisource data and accurate analysis to study and reduce the uncertainty of dealing with various emergency 
problems ${ }^{[7]}$. Only in this way can we adapt to the new characteristics of emergency management modernization and the new technical process of electronic resource intelligent management.

\section{Improving technical support}

During the crisis period, only through reform and innovation can we rise from adversity. In dealing with emergencies such as COVID-19, all libraries should take advantage of various emerging technologies to promote the infrastructure support of electronic resources, enhance the technical support capability of remote services, as well as expand the mode and content of remote services. Libraries should realize the integration of remote services and library intelligent services as well as apply information technologies and methods, such as big data, artificial intelligence, virtual reality technology, knowledge discovery, digital humanities, and knowledge atlas, to create an intelligent service platform as well as enhance service innovation and ability extension. In the pandemic era, grid services and precision services are needed by readers ${ }^{[8]}$, so more emphasis should be on user demand mining and root tracing as well as mine knowledge context; in addition, new social tools should be introduced to enable users to obtain the best experience. The library should also become an example of resource sharing, fully absorb the available resources from the outside with an open and innovative attitude, share cloud technology, create seamless connection between institutions, open related data, strengthen data management and monitoring, as well as improve the technical application and function of the library.

Many libraries have launched mobile library applications, and some libraries are actively using social media channels to create interactive and social spaces with service characteristics. More and more flexible and secure products (including cloud-based products), which attract users, are being developed. In order to give better play to their own advantages, libraries need to integrate the open environment and surpass the open environment under the new technology environment. They also need to have close contact with institutions and communities, assume the role of a hub and intermediary of digital skills and information retrieval services, as well as maintain the mainstream status of cultural information in the whole society.

\section{Promoting collaborative integration and sharing of resources}

Under the background of big data and intelligent emergency, the collaborative integration and sharing of resources is an important content of the library emergency support system. Libraries play an important role in the existing information resource sharing system. Regional document information resource sharing and joint service is an important aspect of China's information service ${ }^{[9]}$. It mainly takes the lead in cross system and cross regional sharing with the document guarantee system of provincial university libraries or carries out collaborative services based on the large database platform to obtain literature contents that have not been ordered by libraries, so as to achieve "openness" and sharing of literature resources to the public. However, the cooperative service mechanism of library information resource sharing is flawed. A wide range of services, such as interlibrary loan and electronic resource sharing, have not been implemented by university libraries across the country ${ }^{[10]}$.

In the post-pandemic era, it is also necessary to establish a system platform for collaborative integration and sharing of information resources based on the electronic resource supply chain, take user needs as the starting point, and establish a multi-party collaborative mechanism, in which libraries, enterprises, institutions, users, and other subjects become a community of interests, constantly carry out technological innovation, and promote the optimization as well as the upgrading of resources. This kind of resource coconstruction and sharing service system, which is highly shared and responsive, gives full play to the advantages of all parties and realizes the integration of resource collection, storage, integration, retrieval, service, supervision, as well as management, can promote the improvement of the overall value of the system. 


\section{Strengthening community integration}

In the face of public health emergencies, libraries should have a sense of social responsibility to provide services for social stability and development. Libraries must strengthen the capacity-building of social linkage and community integration. On the one hand, based on cloud computing, the data resources, platforms, technologies, services, and even the interests and cultures of different libraries and scientific research institutions are integrated to form collaborative governance decentralization. Through cloud services, they can integrate with social knowledge networks and public information platforms, integrate public data with government open data, as well as realize the comprehensive upgrading of the information system ecological chain. On the other hand, libraries should actively meet the diversified needs of the society, support the reading needs of the public, and provide access to public information as well as emergency psychological counseling. The library can provide competitive intelligence service for the scientific and technological innovation of small and medium-sized enterprises as well as provide online learning for residents, job training, information tools, and technology training. By actively participating in the government's information engineering and infrastructure construction, libraries can vigorously popularize information connectivity and digitization. Libraries should also use social media channels to build a virtual community service network and platform, enhance community communication and cooperation, give full play to the role of virtual community in emergency management, as well as mobilize the backbone of the community to participate in resource construction and consulting services.

\section{Providing intelligent management and services}

According to a survey, from the pandemic outbreak in early 2020 to the current pandemic prevention and control stage, various libraries across the country have made efforts to offer several emergency support services, as shown in Table 1.

Table 1. Emergency support services offered by the library

\begin{tabular}{ll}
\hline $\begin{array}{l}\text { "Cloud loan and purchase" as well as other } \\
\text { intelligent services }\end{array}$ & Electronic resources remote services to support teaching and research \\
\hline Online lectures and training & Provide information reference for government decision-making and think tanks \\
\hline $\begin{array}{l}\text { Shuxiang anti-epidemic online reading } \\
\text { service }\end{array}$ & Thematic information collection, integration, and push \\
\hline
\end{tabular}

Emergency service product development Build virtual communities and carry out social services

and service

In the post-pandemic era, anyone can be a library user. Libraries should establish contact and cooperation with everyone. Intelligent management is to manage resources, data, and users through cloud infrastructure. It uses big data to drive the ecological interaction between all parties ${ }^{[11]}$, realize more accurate knowledge analysis and organization, accurately match resources and knowledge services, establish users' action preference and prediction by using the geographic information system (GIS) and user data mining, as well as carry out hierarchical services according to the specific conditions of users. It actively and pertinently pushes information and knowledge services, such as medical treatment as well as learning and scientific research.

The intelligent management and service of the library depends on the ability of librarians. In emergency conditions, libraries should quickly establish a professional online service team, shift from collecting resources to compiling resources, and then to promoting services ${ }^{[12]}$. In emergencies, librarians of library electronic resources should have the ability to effectively communicate with internal and external audiences 
and help users solve practical problems. Therefore, they should pay more attention to the application of internet technology as well as master the theoretical and practical knowledge of electronic resource access, hardware, and software. They should be familiar with relevant laws, digital tools, digital standards, and relevant initiatives ${ }^{[13]}$. They should master more cutting-edge discipline knowledge, develop skills to solve professional and complex problems, and meet the personalized needs of users.

\section{Disclosure statement}

The author declares no conflict of interest.

\section{References}

[1] Yan P, et al., 2011, Research on Connotation and Evaluation Index System of Traffic Emergency Support Capacity. Logistics Technology, 30(6): 19-21.

[2] Zhao F, Analysis Framework and Action Paradigm of Library Public Crisis Governance. Library Development, 1: 1-15.

[3] Wei D, et al., 2020, An Expert's Talk on Library Emergency Service in Major Public Security Emergencies. Library Journal, 39(3): 4-18.

[4] Yuan Q, Chen X, 2020, COVID-19 Response Strategy for "Double First-Class" Construction University Libraries in Wuhan. Library and Information Service, 64(15): 4-11.

[5] Li C, Xin X, 2019, Research on Establishment of Ecosystem Model for Optimal Deployment of Electronic Resources. Library Work and Study, 2019(6): 86-92.

[6] Guo L, Liu C, 2016, Research on Government Emergency Information Resource Planning Based on EA in big Data Environment. Journal of Information, 2016(06): 171-176.

[7] Jiang X, Zhu X, 2020, Think Tank Emergency Intelligence Service Based on Government's Big Data Capabilities: A Case Study of COVID-19 Prevention and Control. Library \& Information, 2020(3): 64-74.

[8] Lv C, 2020, Analysis on the Construction of University Library Emergency Service System under Emergencies - A Case Study of "Double First-Class" University Libraries Responding to COVID-19 Outbreak. Library Work and Study, 2020(12): 116-122.

[9] Song J, Shao F, 2020, Investigation and Analysis of Public Library Emergency Services under Major Security Emergencies. Journal of Academic Library and Information Science, 38(05): 13-20, 37.

[10] Liu P, Tian Z, 2021, Analysis on Intelligent Service Strategies of University Libraries in the Post-Epidemic Era. Library Work and Study, 2021(10): 105-109.

[11] Wang J, et al., 2020, Research on Intelligent Technology Fusion Mechanism of Collaborative Governance of Intelligent Libraries in the Era of Algorithms. Library, 2020(1): 53-58, 87.

[12] Cai Y, Wu Z, 2020, Emergency Guarantee System of University Library Information Resources. Library Journal, 39(05): 43-54.

[13] Fu R, 2020, Application and Analysis of the American Standard of "Core Competence of Electronic Resource Librarians". Library Development, 2020(3): 131-136.

Publisher's note

Bio-Byword Scientific Publishing remains neutral with regard to jurisdictional claims in published maps and institutional affiliations. 